\title{
STATIONARY POINTS OF SET-VALUED CONTRACTIVE AND NONEXPANSIVE MAPPINGS ON ULTRAMETRIC SPACES
}

\author{
MERAJ HOSSEINI*, KOUROSH NOUROUZI* AND DONAL O'REGAN** \\ *Faculty of Mathematics, K. N. Toosi University of Technology, Tehran, Iran \\ E-mails: meraj.hy@gmail.com nourouzi@kntu.ac.ir \\ **School of Mathematics, Statistics and Applied Mathematics \\ National University of Ireland Galway, Ireland \\ E-mail: donal.oregan@nuigalway.ie
}

Abstract. In this paper we show that contractive set-valued mappings on spherically complete ultrametric spaces have stationary (or end) points if they have the approximate stationary point property. We also extend some known fixed point results to nonexpansive set-valued mappings.

Key Words and Phrases: Stationary point, fixed point, approximate stationary point property, ultrametric spaces.

2010 Mathematics Subject Classification: 47H09, 54C60.

\section{REFERENCES}

[1] A. Amini-Harandi, Endpoints of set-valued contractions in metric spaces, Nonlinear Anal., 72(2010), no. 1, 132-134.

[2] D.W. Boyd, J.S. Wong, On nonlinear contractions, Proc. Amer. Math. Soc., 20(1969), 458-464.

[3] N. Dunford, J. Schwartz, Linear Operators, Part I: General Theory, Wiley Interscience, New York, 1957.

[4] W.A. Kirk, N. Shahzad, Some fixed point results in ultrametric spaces, Topology Appl., 159(2012), no. 15, 3327-3334.

[5] C. Petalas, T. Vidalis, A fixed point theorem in non-Archimedean vector spacs, Proc. Amer. Math. Soc., 118(1993), no. 3, 819-821.

[6] A. Petruşel, I.A. Rus, M.A. Şerban, Basic problems of the metric fixed point theory and the relevance of a metric fixed point theorem for a multivalued operator, J. Nonlinear Convex Anal., 15(2014), no. 3, 493-513.

[7] A.C.M. van Rooij, Non-Archimedean Functional Analysis, Marcel Dekker, New York, 1978.

[8] I.A. Rus, Strict fixed point theory, Fixed Point Theory, 4(2003), no. 2, 177-183.

[9] K. Włodarczyk, R. Plebaniak, Endpoint theory for set-valued nonlinear asymptotic contractions with respect to generalized pseudodistances in uniform spaces, J. Math. Anal. Appl., 339(2008), no. 1, 344-358.

[10] K. Włodarczyk, D. Klim, R. Plebaniak, Existence and uniqueness of endpoints of closed setvalued asymptotic contractions in metric spaces, J. Math. Anal. Appl., 328(2007), no. 1, 46-57.

Received: September 2, 2015; Accepted: October 8, 2015. 
